\title{
Cirurgia de Revascularização na Fase Aguda do Infarto do Miocárdio. Análise dos Fatores Pré-operatórios Preditores de Mortalidade
}

\author{
Coronary Artery Bypass Grafting in Acute Myocardial Infarction. Analysis of \\ Preoperative Predictors of Mortality
}

Renata Teixeira Ladeira, Fabio Biscegli Jatene, Rosangela Monteiro, Simone Pereira Zucato, Luciano Moreira Baracioli, Alexandre Ciappina Hueb, Luis Alberto Oliveira Dallan, Luiz Boro Puig,

Sérgio Almeida Oliveira, José Carlos Nicolau

Instituto do Coração do Hospital das Clínicas - FMUSP - São Paulo, SP

\section{Objetivo}

Analisar os fatores pré-operatórios preditores de mortalidade, em pacientes submetidos à RM nos primeiros 30 dias após infarto agudo do miocárdio (IAM).

\section{MÉtodos}

Entre 3/1998 e 7/2002, foram incluídos, consecutiva e prospectivamente, em um banco de dados, 753 pacientes com IAM, sendo que 135 (17,9\%) foram submetidos à revascularização miocárdica (RM) isolada e incluídos neste estudo. Estudaram-se os seguintes fatores prognósticos, através de análise multivariada: idade, sexo, diabete, história de IAM, RM ou angioplastia (ATC), localização do IAM, IAM $Q$, uso de fibrinolítico, intervalo entre o IAM e a cirurgia, presença de complicações no pré-operatório.

\section{Resultados}

A mortalidade hospitalar global foi de $6,7 \%$, variando de $12,5 \%$ nos pacientes portadores de complicações pré-operatórias a 1,4\% naqueles sem complicações. Tiveram correlação estatisticamente significante com a mortalidade pós-operatória apenas história prévia de angioplastia $(p=0,037)$ e choque cardiogênico $(p=0,002)$. Em contrapartida, o uso de trombolítico na abordagem inicial do IAM apresentou correlação negativa com a mortalidade $(p=0,035)$.

\section{Conclusão}

A RM na fase aguda do IAM é um procedimento que apresenta mortalidade cirúrgica distinta, na dependência da condição clínica pré-operatória do paciente. Dentre os fatores analisados, a presença de choque cardiogênico préoperatório e história de angioplastia prévia determinaram pior prognóstico neste grupo de pacientes.

\section{Palavras-chave}

Mortalidade, cirurgia, infarto agudo do miocárdio.

\section{Objective}

To assess preoperative predictors of mortality in patients undergoing coronary artery bypass grafting (CABG) within the first 30 days of acute myocardial infarction (AMI).

\section{Methods}

Between March 1998 and July 2002, 753 AMI patients were consecutively and prospectively entered into a database, 135 (17.9\%) of whom underwent isolated CABG and were enrolled in this study. The following prognostic factors were assessed by multivariate analysis: age, gender, diabetes, history of previous AMI, $C A B G$ or coronary angioplasty (PTCA), anterior infarct location, Q-wave AMI, the use of fibrinolytics, elapsed time from AMI to the procedure, and presence of complications in the preoperative period.

\section{RESULTS}

Overall in-hospital mortality was $6.7 \%$, ranging from $12.5 \%$ in patients with preoperative complications to $1.4 \%$ in those with no complications. Only history of previous angioplasty $(p=0.037)$ and cardiogenic shock $(p=0.002)$ showed a statistically significant correlation with postoperative mortality. The use of thrombolytics, on the other hand, in the initial management of AMI showed a negative correlation with mortality $(p=0.035)$.

\section{Conclusion}

CABG in the acute phase of $\mathrm{MI}$ is associated with distinct operative mortality, depending on the patient's preoperative clinical condition. Among those factors analyzed, preoperative cardiogenic shock and history of previous angioplasty were predictive of worse prognosis in this group of patients.

\section{KEY WORDS}

Mortality, surgery, acute myocardial infarction.

Correspondência: Renata Teixeira Ladeira - Rua dos Caetés, 737/205 - São Paulo, SP - 05016-081 
Desde a década de 1970 , têm sido realizados trabalhos buscando determinar o intervalo de tempo ideal para realização da cirurgia de revascularização miocárdica (RM) após o infarto agudo do miocárdio (IAM) com menor risco cirúrgico ${ }^{1-7}$. Entretanto, ainda não existe consenso na literatura sobre o momento mais apropriado para realização da RM após $\mathrm{IAM}^{6}$. Alguns grupos aguardam um período de até 30 dias após o IAM, para a realização da operação, buscando, desta forma, evitar as possíveis complicações que poderiam advir no período de recuperação do infarto ${ }^{6}$.

Inicialmente, a cirurgia era usada como forma de recanalização, sendo realizada o mais precoce após o IAM, com objetivo de redução da área infartada, porém com morbi-mortalidade cirúrgica elevada ${ }^{1-4}$. A partir da década de 1980, a RM como forma de recanalização após IAM, tem sido cada vez menos utilizada, já que dispomos de outras técnicas, como: fibrinolíticos e procedimentos percutâneos ${ }^{8,9}$. Assim, a cirurgia fica reservada, nesta fase, para os pacientes em cuja recanalização as técnicas acima falharam ou evoluíram com angina persistente ou instabilidade hemodinâmica ${ }^{10}$. Tem-se um outro grupo de pacientes em que a recanalização é obtida com sucesso; porém, o doente possui obstruções coronarianas residuais importantes, que necessitam de intervenção cirúrgica ${ }^{11}$. Em pacientes estáveis clinicamente, a mortalidade cirúrgica é de 2 a $4 \%$, bastante semelhante à de pacientes revascularizados eletivamente ${ }^{10,11}$. Entretanto, nos pacientes com complicações clínicas ou mecânicas, a mortalidade sobe para níveis de 6 a 26\% ${ }^{12-14}$.

O objetivo deste estudo é analisar os fatores préoperatórios preditores de mortalidade, em pacientes submetidos à RM, nos primeiros 30 dias após IAM.

\section{MÉTOdOS}

No período de 3/1998 a 7/2002, 753 pacientes foram admitidos na unidade coronariana, com diagnóstico de IAM, sendo incluídos consecutiva e prospectivamente em um banco de dados específico. Destes, 145 (19,3\%) foram submetidos a RM cirúrgica, na fase aguda e subaguda do IAM.

A RM foi um procedimento isolado, em 135 (93,1\%) pacientes, constituindo os pacientes analisados neste estudo. Excluíram-se desta análise 10 (6,9\%) pacientes que foram submetidos a procedimentos associados à RM (como troca ou plástica valvar) ou, ainda, que apresentaram complicações mecânicas secundárias ao IAM, como ruptura do septo interventricular .

O grupo foi composto por pacientes que apresentavam lesões uni, bi ou triarteriais maiores que $70 \%$ do lúmen coronariano, com função ventricular preservada ou não. A idade dos pacientes, no momento da operação, variou de 29 a 94 anos, com média de $62,3 \pm 11,2$ anos, sendo $102(75,6 \%)$ do sexo masculino. Quarenta $(29,6 \%)$ pacientes apresentavam diabete melito tipo II, $32(23,7 \%)$ tinham histórico de IAM prévio, $11(8,1 \%)$ já haviam sido submetidos à revascularização miocárdica anteriormente e $9(6,7 \%)$ à angioplastia coronariana.

Quanto à localização do IAM, a parede anterior do coração foi acometida em $46 \%$ dos pacientes, a inferior em $43 \%$ e outras regiões em $11 \%$ dos casos. O IAM ocorreu com onda Q em $86(63,7 \%)$ pacientes. Trinta e nove $(28,9 \%)$ pacientes fizerem uso de fibrinolítico, principalmente estreptoquinase, previamente à operação.

Foram avaliadas as seguintes variáveis pré-operatórias, como prognósticas para revascularização miocárdica na fase aguda do IAM: idade, sexo, história de diabete melito, infarto prévio, cirurgia de revascularização miocárdica ou angioplastia prévia (ambas anteriores ao IAM em estudo), IAM com ou sem onda $Q$, infarto de parede anterior, uso de fibrinolítico, intervalo entre o IAM e a RM, presença de complicações pré-operatórias [isquemia recorrente, insuficiência cardíaca congestiva (ICC), choque cardiogênico (CC), reinfarto, taquicardia ventricular sustentada (TVS), fibrilação ventricular $(\mathrm{FV})$ ]. A incidência destas complicaçốes encontra-se na tabela 1. Apesar de a fração de ejeção ventricular esquerda ser um fator prognóstico importante, em pacientes submetidos à cirurgia de RM, optou-se, na presente análise, por trabalhar especificamente com dados clínicos, facilmente obtidos em qualquer paciente, mesmo naqueles que realizam o procedimento de forma urgente ou emergencial. Assim, foram incluídos ICC e CC, que sabidamente apresentam excelente correlação

\section{Tabela 1 - Incidência de complicações pré- operatórias}

$\begin{array}{lc}\text { Complicações pré-operatórias } & \mathbf{n}(\%) \\ \text { Isquemia recorrente } & 45(33,3 \%) \\ \text { Insuficiência cardíaca } & 21(15,6 \%) \\ \text { Choque cardiogênico } & 12(8,9 \%) \\ \text { Reinfarto } & 9(6,7 \%) \\ \text { Taquicardia ventricular sustentada } & 8(5,9 \%) \\ \text { Fibrilação ventricular } & 5(3,7 \%)\end{array}$

com fração de ejeção.

A mediana do intervalo entre o IAM e a RM foi de 10,6 dias, com média de 11,6 dias (desvio padrão de 6,4 dias). Em média, foram empregados 3,0 enxertos/ paciente, sendo 184 enxertos arteriais (1,4 enxerto arterial/paciente) e 219 enxertos venosos (1,6 enxerto venoso/paciente), evidenciando que a RM foi completa. A artéria torácica interna esquerda foi utilizada como enxerto, em 117 (63,6\%) pacientes; a artéria torácica interna direita, em 32 (17,7\%); a artéria radial esquerda, em 33 (17,9\%); e outros enxertos arteriais, em $2(1,1 \%)$ casos.

Em relação à região revascularizada, a parede anterior foi revascularizada com $204(50,6 \%)$ enxertos, a lateral com $114(28,3 \%)$ enxertos e a inferior com 85 $(21,1 \%)$.

A análise estatística foi realizada utilizando-se o programa Systat. Os dados foram submetidos inicialmente às análises univariadas com teste de Fisher e qui-quadrado para variáveis categóricas e teste de MannWhitney para variáveis contínuas. Análise multivariada dos fatores prognósticos foi feita através de regressão logística stepwise. Foram considerados significativos os valores de $p<0,05$.

\section{Resultados}

Foram observados $9(6,7 \%)$ óbitos, no período hospitalar. Entretanto, quando as complicações clínicas pré-operatórias 
foram agrupadas como uma variável única, observamos 8 (12,5\%) óbitos, no grupo de pacientes em que elas estavam presentes. Em contrapartida, houve apenas $1(1,4 \%)$ óbito dentre os pacientes que não apresentavam complicações clínicas no pré-operatório (odds ratio $10, p=0,013$, fig. $1)$.

$\mathrm{Na}$ análise univariada, apenas as variáveis choque cardiogênico, fibrilação ventricular e história de angioplastia apresentaram correlação com mortalidade. As demais variáveis analisadas não apresentaram correlação estatisticamente significante com a mortalidade (tab. 2).
Dos 12 pacientes com choque cardiogênico, 5 (41,7\%) foram a óbito após a cirurgia $(\mathrm{OR}=21,2 ; \mathrm{p}<0,001)$. Foram observados 2 (40\%) óbitos dentre os 5 pacientes que apresentavam fibrilação ventricular pré-operatória $(\mathrm{OR}=$ $11,7 ; p=0,002)$ e $2(22,2 \%)$ óbitos dentre os 9 pacientes com história de angioplastia prévia $(\mathrm{OR}=4,8 ; \mathrm{p}=0,053)$.

No modelo final da análise multivariada, apenas história prévia de angioplastia $(p=0,037)$ e choque cardiogênico $(p=0,002)$ mantiveram significância estatística com a variável óbito. Em contrapartida, o uso de fibrinolítico na abordagem inicial do IAM apresentou correlação negativa

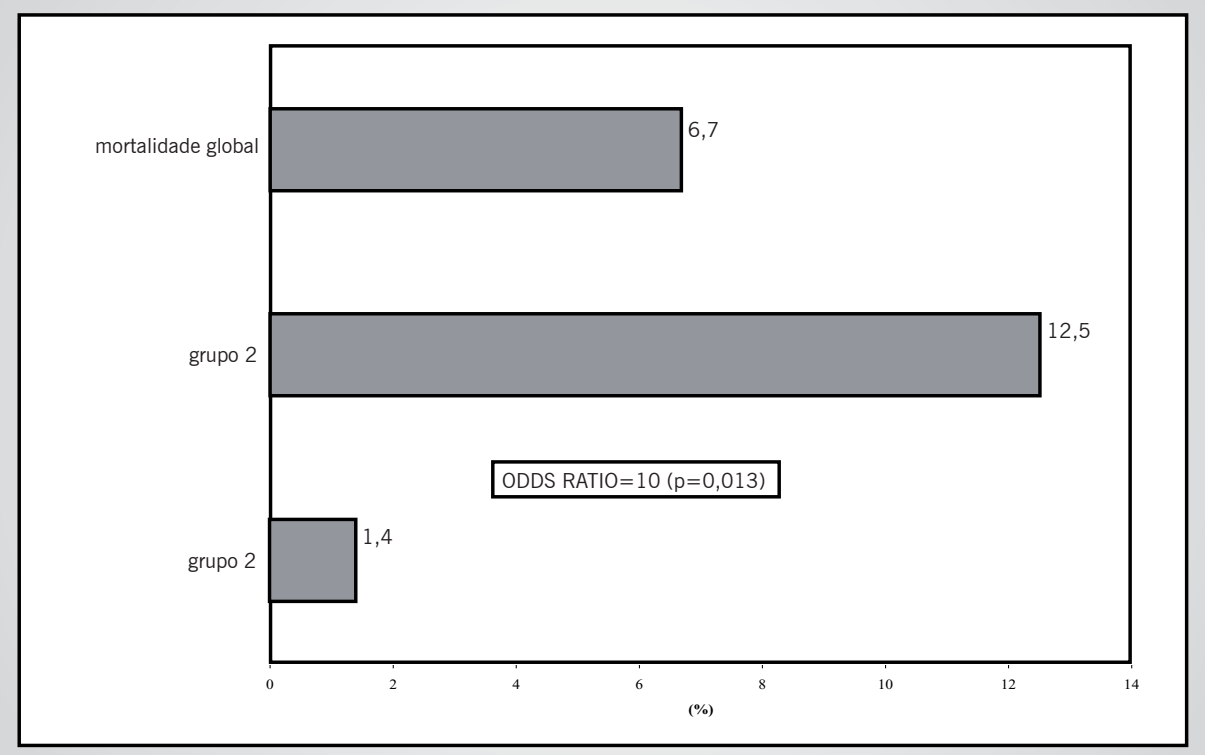

Fig. 1 - Mortalidade hospitalar dos pacientes com IAM submetidos à RM. Grupo 1 (sem complicações clínicas pré-operatórias) e grupo 2 (com complicações clínicas pré-operatórias).

\begin{tabular}{|c|c|}
\hline \multicolumn{2}{|c|}{$\begin{array}{c}\text { Tabela } 2 \text { - Análise univariada dos fatores pré } \\
\text { operatórios. }\end{array}$} \\
\hline Fatores prognósticos pré-operatórios & $\mathrm{p}$ \\
\hline Idade & $p=0,167$ \\
\hline Sexo & $p=0,872$ \\
\hline Diabete melito & $p=0,314$ \\
\hline Angioplastia prévia & $p=0,053$ \\
\hline Revascularização prévia & $p=0,737$ \\
\hline IAM prévio & $p=0,482$ \\
\hline IAM com onda $Q$ & $p=0,363$ \\
\hline IAM anterior & $p=0,926$ \\
\hline IAM inferior & $p=0,430$ \\
\hline Uso de fibrinolítico & $p=0,257$ \\
\hline Intervalo entre IAM e RM & $p=0,379$ \\
\hline Isquemia recorrente & $p=0,143$ \\
\hline ICC & $p=0,128$ \\
\hline Choque cardiogênico & $p<0,001$ \\
\hline Taquicardia ventricular sustentada & $p=0,495$ \\
\hline Fibrilação ventricular & $p=0,002$ \\
\hline Reinfarto & $p=0,407$ \\
\hline $\begin{array}{l}\text { IAM - infarto agudo do miocárdio; RM } \\
\text { miocárdica; ICC - insuficiência cardía }\end{array}$ & $\begin{array}{l}\text { larização } \\
\text { tiva. }\end{array}$ \\
\hline
\end{tabular}

com a mortalidade $(p=0,035)$, ratificando a importância da recanalização na fase aguda do IAM.

\section{Discussão}

A indicação de RM na fase aguda do IAM continua sendo tema controverso, sem que haja consenso na literatura sobre o momento ideal de sua indicação ${ }^{15-17}$. Teoricamente, haveria vantagens em esperar a cicatrização do IAM (4-6 semanas), quando haveria completa recuperação do miocárdio atordoado, sem risco de injúria miocárdica após reperfusão, que pode, inclusive, gerar infarto hemorrágico ${ }^{15,18}$. Entretanto, devem-se pesar os riscos desta espera, como o de isquemia recorrente, com chance de reinfarto, devido às lesões presentes; expansão da área do IAM, com remodelamento ventricular, podendo gerar aneurisma e aumento importante dos custos com internações prolongadas ${ }^{15,17,19}$.

Há um grupo de pacientes no qual a RM é uma urgência, sendo necessária a indicação, como nos casos de complicações mecânicas associadas ao IAM $^{20}$, ou pacientes com choque cardiogênico e com lesão de tronco de artéria coronária esquerda ou triarteriais ${ }^{21,22}$. Entretanto, há dúvidas de quando operar os pacientes que, a despeito de boa evolução clínica pós-IAM, 
apresentam lesões multiarteriais ou mesmo lesões isoladas, de características particulares, que necessitam de $\mathrm{RM}^{23-25}$

Referente aos aspectos cirúrgicos, há particularidades na RM após IAM. Braxton e cols. mostraram o uso de 2,4 enxertos/pacientes no grupo com IAM e 2,6 no grupo controle (sem IAM); porém, artéria torácica interna esquerda (ATIE) foi usada em $43 \%$ dos pacientes operados com <48hs, $72 \%$ nos operados entre 3-5 dias, 92\% entre 6-42 dias e $82 \%$ nos controles ${ }^{5}$. Johanes e cols., apesar de descreverem como objetivo a RM completa e uso da ATIE em todos os pacientes, aplicaram ATIE em $87 \%$ dos pacientes com IAM, versus $97 \%$ dos eletivos $(p=0,008)^{10}$. De forma semelhante, em nossos pacientes, a média de enxertos usados foi de 3,0, refletindo a importância da revascularização completa, no sentido de prevenir complicações como isquemia recorrente ou reinfarto no pós-operatório, que podem prejudicar a evolução dos pacientes. Além disso, buscamos sempre que possível empregar a ATIE $(63,6 \%)^{26,27}$, considerando suas vantagens já bem estabelecidas na literatura

Em 1994, Sintek e cols. publicaram os resultados referentes a 530 pacientes com IAM que, após receberem tratamento com fibrinolítico e serem estratificados, tiveram indicação cirúrgica de RM. Destes, 23 pacientes foram operados dentro das primeiras $24 \mathrm{~h}$, com mortalidade hospitalar de 4,4\%; 30 entre 24 e 72 h, sem óbitos; 193 entre 3 e 7 dias apresentaram mortalidade de $2,1 \%$ e 284 entre 7 dias e 1 mês, com mortalidade de $1,4 \%$. Comparativamente, 1.645 pacientes operados sem IAM prévio tiveram mortalidade cirúrgica de 1,9\%. Foram empregados 2,9 enxertos por paciente. Houve necessidade de uso de balão intra-aórtico, em $6,7 \%$ dos pacientes com IAM e apenas $2,6 \%$ no grupo-controle ${ }^{17}$.

Lee e cols. avaliaram retrospectivamente 32.099 pacientes submetidos à RM após IAM transmural em 33 hospitais no estado de New York, EUA. Concluíram que a RM realizada no período de 3 dias após o IAM pode trazer risco adicional à mortalidade hospitalar, independente de outros fatores de risco cirúrgicos que foram incluídos em análise multivariada. A mortalidade cirúrgica foi variável em diferentes intervalos de tempo entre o IAM e a operação, sendo de $14,2 \% ; 13,8 \%$; $7,9 \% ; 3,8 \% ; 2,9 \%$ e $2,7 \%$ para cirurgias realizadas com menos de $6 \mathrm{~h}, 6 \mathrm{~h}$ a 1 dia, 1 a 3 dias, 4 a 7 dias, 7 a 14 dias e 15 dias ou mais, respectivamente. E, assim, recomendam que, se não houver indicação para cirurgia de emergência, deve-se aguardar um período de 3 dias para se realizar a RM pois, após este período, os índices de mortalidade são similares ${ }^{6}$. Os dados no nosso estudo corroboram esta recomendação, já que a mediana do intervalo entre o IAM e a RM foi de 10,6 dias. Podemos dividir nossa experiência em 3 fases, que nos permitiram observar uma tendência de realização mais precoce da cirurgia. Nos primeiros pacientes, o intervalo médio foi de 13,6 dias; no grupo intermediário, de 12,3 dias; e mais recentemente, os pacientes têm sido encaminhados para cirurgia, em 8,2 dias.

Inúmeros estudos tentam identificar qual grupo de pacientes teria maior risco cirúrgico no pós-IAM ${ }^{9,10,11,16,22,26}$. Dados do National Registry of Myocardial Infarction 2, que avaliou 71.774 pacientes com IAM submetidos à RM, apontam como fatores de risco para mortalidade cirúrgica as seguintes variáveis: sexo feminino, IAM com onda $Q$, idade $>65$ anos, história de ICC e diabete melito, cirurgia de RM prévia e classificação de Killip do IAM $^{11}$.

Applebaum e cols. ${ }^{28}$, analisando 1.726 pacientes submetidos a RM, num período de 6 anos, sendo que 406 foram operados dentro dos primeiros 30 dias pósIAM, observaram mortalidade hospitalar de 2,4\%. No subgrupo de pacientes com história de IAM recente, esta mortalidade foi de $6,7 \%$, enquanto que dentre aqueles sem história de IAM prévio esta foi de 1,1\%. Na análise multivariada, 3 fatores mostraram-se significantemente associados a aumento da mortalidade hospitalar, sendo eles fração de ejeção inferior a 30\% ( $p<0,0001)$, choque cardiogênico pré-operatório $(p=0,0005)$ e idade maior que 70 anos $(p=0,004)$. No seguimento realizado em $90 \%$ dos pacientes, num tempo médio de $35 \pm 21$ meses, $88 \%$ estavam vivos ao final de 3 anos e $84 \%$ em 5 anos após cirurgia. Assim, concluiu-se neste trabalho que pacientes com menos de 70 anos, com fração de ejeção superior a $30 \%$ e que não estão em choque cardiogênico podem ser operados em qualquer momento após IAM, sem risco aumentado ${ }^{28}$. Quanto à fração de ejeção, não incluímos esta variável em nosso estudo; entretanto, há correlação nítida entre baixa fração de ejeção e evolução dos pacientes para choque cardiogênico. A idade não foi incluída em nosso modelo como uma variável categórica e sim como variável contínua, não apresentando associação com mortalidade. Em nossa análise, o choque cardiogênico também foi um importante fator preditor de mortalidade.

No estudo SHOCK, estudou-se a RM precoce em 302 pacientes com IAM complicado por choque cardiogênico; destes, 152 foram randomizados para revascularização de emergência e 150 para estabilização clínica. A revascularização foi feita, dentro das primeiras $6 \mathrm{~h}$ da randomização, tanto por cirurgia cardíaca (57 pacientes) como por procedimento hemodinâmico (75 pacientes). A mortalidade global em 30 dias não diferiu significantemente $(p=0,11)$ entre os grupos de tratamento clínico e de revascularização (56\% e $46,7 \%$, respectivamente). Entretanto, a mortalidade em 6 meses foi menor no grupo revascularizado que no clínico (50,3\% versus $63,1 \%, p=0,027)$. Concluiu-se que a revascularização precoce deve ser fortemente considerada, para pacientes com IAM complicado por choque cardiogênico ${ }^{21}$.

Há também um outro aspecto a ser ressaltado: nossa mortalidade global foi de $6,7 \%$, idêntica à observada pelo grupo de Applebaum ${ }^{28}$. Entretanto, num subgrupo de pacientes de nossa casuística, sem complicações clínicas pré-operatórias, a mortalidade foi de apenas $1,4 \%$, comparável e até mesmo inferior à mortalidade em cirurgias eletivas.

Assim, apesar de décadas de experiência, encontramos trabalhos que mostram aumento da mortalidade dos pacientes operados precocemente após $\operatorname{IAM}^{5,6,15,16}$ e outros que relatam ser segura a RM em qualquer período após o $\mathrm{IAM}^{17,25,29,30}$. Em nosso estudo, não houve relação da mortalidade e o intervalo entre IAM e RM. Na análise multivariada o que determinou maior mortalidade cirúrgica foi a presença de choque cardiogênico e história previa de angioplastia. Realmente, há maior mortalidade nos pacientes em que a angioplastia é realizada na mesma internação em que ocorre a RM; entretanto, ainda não está bem definida a relação entre história prévia 
de angioplastia e mortalidade cirúrgica, necessitando assim outros estudos para melhor esclarecimento desta questão ${ }^{31}$. Pode haver variáveis associadas à história prévia de angioplastia, como coronariopatia mais difusa, pior função do ventrículo esquerdo, dentre outras, que não foram incluídas na nossa análise e que podem confundir esta conclusão.

Apesar do receio de aumento de sangramento durante o procedimento cirúrgico e no pós-operatório de pacientes que foram submetidos à fibrinólise farmacológica, alguns grupos têm mostrado que a RM pode ser realizada precocemente nestes pacientes, com baixa morbimortalidade ${ }^{32}$. Como visto em nossa casuística, o uso de fibrinolítico foi benéfico, sendo um fator protetor durante a cirurgia, já que a recanalização na fase aguda propicia maior estabilidade clínica a longo prazo.

Assim, concluímos que a RM na fase aguda do IAM é um procedimento que apresenta mortalidade cirúrgica aceitável. Dentre os fatores analisados, a presença de choque cardiogênico pré-operatório e história de angioplastia prévia determinaram pior prognóstico neste grupo de pacientes.

\section{Potencial Conflito de Interesses}

Declaro não haver conflitos de interesses pertinentes.

\section{REFERÊNCIAS}

1. Hill JD, Kerth WJ, Kelly JJ, et al. Emergency aortocoronary bypass for impending or extending myocardial infarction. Circulatin 1971; 43(5 Suppl): |-105-10

2. Dawson JT, Hall RJ, Halman GL, Cooley DA. Mortality in patients undergoing coronary artery bypass surgery after myocardial infarction. Am J Cardiol 1974; 33:483-6.

3. Berg R Jr, Kendall, Duvoisin GE, Ganji JH, Rudy LW, Everhart FJ. Acute myocardial infarction: a surgical emergency. J Thorac Cardiovasc Surg 1975; 70: 432-9.

4. DeWood MA, Spores J, Berg R Jr, et al. Acute myocardial infarction: a decade of experience with surgical reperfusion in 701 patients. Circulation 1983; 68(suppl):II-8-16

5. Braxton JH, Hammond GL, Letson GV, et al. Optimal timing of coronary artery bypass graft surgery after acute myocardial infarction. Circulation 1995; 92(Suppl 9): II-66-8.

6. Lee $\mathrm{DC}, \mathrm{Oz} \mathrm{MC}$, Weinberg AD, Ting W. Appropriate timing of surgical intervention after transmural acute myocardial infarction. J Thorac Cardiovas Surg. 2003; 125:115-20

7. Ladeira RT, Jatene FB, Zucato SP, et al. Análise da mortalidade de pacientes com infarto agudo do miocárdio submetidos precocemente à cirurgia de revascularização miocárdica. Arq Bras Cardiol 2003; 81 (supl. III): 53. Tema livre apresentado no $58^{\circ}$ Congresso da Sociedade Brasileira de Cardiologia.

8. Chesebro JH, Knatterud G, Roberts R, et al. Thrombolysis in Myocardial Infarction (TIMI) Trial, Phase I: a comparison between intravenous tissue plasminogen activator and intravenous streptokinase. Clinical findings through hospital discharge. Circulation 1987; 76: 142-54.

9. The Global Use of Strategies to Open Occluded Coronary Arteries in Acute Coronary Syndromes (GUSTO IIb) Angioplasty Substudy Investigators. A clinical trial comparing primary coronary angioplasty with tissue plasminogen activator for acute myocardial infarction. $\mathrm{N}$ Engl J Med 1997; 336:1621-8.

10. Albes JM, Gross M, Franke U, et al. Revascularization during acute myocardial infarction: risks and benefits revisited. Ann Thorac Surg 2002; 74: 102-8.

11. Zaroff JG, diTommaso DG, Barron HV for the Investigators in the National Registry of Muocardial Infarction 2. A risk model derived from the national registry of myocardial infarction 2 database for predicting mortality after coronary artery bypass grafting during acute myocardial infarction. Am J Cardiol 2002; 90:1-4.

12. Kennedy J, Ivey T, Misbach G, et al. Coronary artery bypass for recent infarction: predictors of mortality. Circulation 1988;78:I-73-I-78.

13. Kereiakes D, Topol E, George B and the TAMI Study Group. Favorable early and long-term prognosis following coronary bypass surgery therapy for myocardial infarction: results of a multicenter trial. Am Heart J 1989;118:199-207.
14. Every NR, Maynard C, Cochran RP, Martin J, Weaver WD. Characteristics, management, and outcome of patients with acute myocardial infarction treated with bypass surgery. Myocardial Infarction Triage and Intervention Investigators. Circulation 1996;94:1181-6.

15. Deeik RK, Schmitt TM, Ihrig TG, Sugimoto JT, Nebraska . Appropriate timing of elective coronary artery bypass graft surgery following acute myocardial infarction. Am J Surg 1998; 176:581-5.

16. Creswell LL, Moulton MJ, Cox JL, Rosenbloom M. Revascularization after acute myocardial infarction. Ann Thorac Surg 1995; 60:1926.

17. Sintek CF, Pfeffer TA, Khonsari S. Surgical revascularization after acute myocardial infarction. Does timing make a difference? J Thorac Cardiovas Surg. 1994; 107(5): 1317-22.

18. Roberts CS, Schoen FJ, Kloner RA. Effect of coronary reperfusion on myocardial hemorrhage and infarct healing. Am J Cardiol 1983; 52:610-14.

19. Weiss JL, Marino N, Shapio EP. Myocardial infarct expansion: recognition , significance and pathology. Am J Cardiol 1991; 68: 35-40.

20. Bolooki H. Surgical treatment of complications of acute myocardial infarction. JAMA 1990; 263:1237-40.

21. Hochman JS, Sleeper LA, Webb JG, et al . Early revascularization in acute myocardial infarction complicated by cardiogenic shock. N Eng J Med 1999;341: 625-34.

22. Berger PB, Tuttle RH, Holmes DR, et al (for the GUSTO I investigation). One-year survival among patients with acute myocardial infarction complicated by cardiogenic shock, and its relation to early revascularization. Circulation 1999; 99:873-8.

23. Naunheim K, Kesler K, Kanter K, et al. Coronary artery bypass for recent infarction: predictors of mortality. Circulation 1988; 78:I-122I-8.

24. Gersh B, Chesebro J, Braunwald E, et al. Coronary artery bypass surgery after thrombolitic therapy in the thrombolysis in myocardial infarction trial, phase II (TIMI II). J Am Coll Cardiol 1995; 25:395402

25. Jatene FB, Nicolau JC, Hueb AC, Atik FA, et al. Fatores prognósticos da revascularização na fase aguda do infarto agudo do miocárdio. Rev Bras Cir Cardiovasc 2001;16(3):195-202.

26. Loop FD, Lytle BW, Cosgrove DM, Golding LA, Taylor PC, Stewart RW. Free (aorta-coronary) internal mammary artery graft. Late results. J Thorac Cardiovasc Surg. 1986; 92:827-31. 
27. Dabal RJ, Goss JR, Maynard C, Aldea GS. The effect of left internal mammary artery utilization on short-term outcomes after coronary revascularization. Ann Thorac Surg. 2003; 76:464-70.

28. Applebaum R, House R, Rademaker A, et al. Coronary artery bypass grafting within thirty days of acute myocardial infarction. J Thorac Cardiovasc Surg 1991;102: 745-52.

29. Kaul TK, Fields BL, Riggins SL, Dacumos GC, Wyatt DA, Jones CR. Coronary artery bypass grafting within 30 days of an acute myocardial infarction. Ann Thorac Surg 1995; 59:1169-76.
30. Stone WG, Brodie BR, Griffin JJ, et al (for the Primary Angioplasty in Myocardial Infarction). Role of cardiac surgery in the hospital phase management of patients treated with primary angioplasty for acute myocardial infarction. Am J Cardiol 2000; 85:1292-6.

31. Jones RH, Hannan EL, Hammermeister KE, et al. Indentification of preoperative variables needed for risk adjustment of short-term mortality after coronary artery bypass graft surgery. J Am Coll Cardiol 1996; 28:1478-87.

32. Petrovich JA, Schneider JA, Taylor GJ, Mikell FL, Batchelder JE, Moses $\mathrm{HW}$, et al. Early and late results of operation after thrombolytic therapy for acute myocardial infarction. J Thorac Cardiovasc Surg. 1986; 92(5):853-8 